\title{
Mid-term durability of the Trifecta bioprosthesis for aortic valve replacement
}

\author{
Amedeo Anselmi, MD, PhD, Vito Giovanni Ruggieri, MD, PhD, Bernard Lelong, MD, \\ Erwan Flecher, MD, PhD, Hervé Corbineau, MD, Thierry Langanay, MD, \\ Jean-Philippe Verhoye, MD, PhD, and Alain Leguerrier, MD
}

\begin{abstract}
Objective: To clarify the mid-term durability of the Trifecta bioprosthesis for aortic valve replacement (AVR).

Methods: We retrospectively analyzed the prospectively collected data of 824 consecutive implants of the Trifecta valve at a single institution. A $100 \%$ complete follow-up was available (average duration, $2.2 \pm 1.3$ years; range, 0.03-6.9 years; 1747.6 patient-years). Echocardiography data at discharge were recorded prospectively.
\end{abstract}

Results: Operative mortality was $3.8 \% ; 2.7 \%$ in patients receiving isolated AVR. There were 5 valve-related early reoperations, including 1 for infective prosthetic endocarditis and 4 for nonstructural valve dysfunction. The global rate of severe patient-prosthesis mismatch was $1.26 \%$. Overall 5-year survival was $74.9 \%$, and freedom from valve-related death was $97.8 \%$. The majority of deaths attributed to the valve were due to unknown causes. We observed 6 SVD events at $3.4 \pm 1.6$ years after surgery. At 5 years, the actuarial freedom from SVD was $98 \% \pm 0.9 \%(\mathrm{n}=6)$, freedom from reintervention for SVD was $98 \% \pm 0.9 \%$ ( $\mathrm{n}=5$, including 2 transcatheter valve-in-valve), and freedom from open reoperation for SVD was $98.9 \% \pm 0.6 \%$. The 5-year freedom from prosthetic endocarditis was $97.7 \% \pm 0.7 \%(\mathrm{n}=12,6$ requiring reoperation $)$. There was 1 case of late NSVD (5-year freedom, 99.8\% $\pm 0.2 \%$ ). Freedom from hemorrhagic events was $98.6 \% \pm 0.5 \%$ ( $86 \%$ occurring in patients on anticoagulants); there were no thromboembolic events at follow-up.

Conclusions: The Trifecta bioprosthesis is a reliable device for AVR. We confirm excellent immediate hemodynamic properties and a very low rate of patientprosthesis mismatch. The absolute number of SVD cases observed remains limited; nevertheless, their timing, pathological characteristics, and clinical presentation mandate continued follow-up. (J Thorac Cardiovasc Surg 2017;153:21-8)

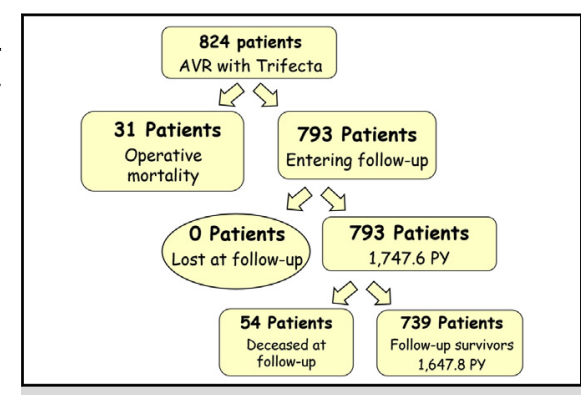

Study workflow and 5-year freedom from valverelated adverse events.

\section{Central Message}

The Trifecta bioprosthesis is a reliable device for aortic valve replacement. Continued surveillance for SVD events is required.

\section{Perspective}

Durability is a pivotal characteristic for modern bioprostheses. In the present mid-term followup of 824 implants, the Trifecta valve showed excellent hemodynamic properties and consistent durability. Few SVD events were observed, characterized by peculiar timing, pathophysiology, and clinical presentation. Continued follow-up is required.

See Editorial Commentary page 29
Bioprostheses are increasingly used for aortic valve replacement (AVR), driven by the continuous improvements in durability and hemodynamic performance over the last several decades. The St. Jude Trifecta bioprosthesis (St. Jude Medical, St. Paul, Minn), a latest-generation stented bioprosthesis, was introduced into clinical practice

\footnotetext{
From the Division of Thoracic and Cardiovascular Surgery, Pontchaillou University Hospital, Rennes, France.

Received for publication Aug 19, 2015; revisions received July 9, 2016; accepted for publication July 30, 2016; available ahead of print Sept 30, 2016.

Address for reprints: Amedeo Anselmi, MD, PhD, Division of Thoracic and Cardiovascular Surgery, Pontchaillou University Hospital, 2 rue Henri Le Guilloux, 35033 Rennes, France (E-mail: amedeo.anselmi@alice.it). $0022-5223 / \$ 36.00$

Copyright (c) 2016 by The American Association for Thoracic Surgery http://dx.doi.org/10.1016/j.jtcvs.2016.07.080
}

in 2007. This device is characterized by a bovine pericardial sheet mounted outside the stent frame. This feature, along with the supra-annular design, the ethanol-based anticalcification treatment (proprietary Linx process ${ }^{1}$ ), and the material properties of the titanium stent, are intended to optimize the device's functional behavior and durability. The stent is polyester-covered and concealed by bovine and porcine pericardium to minimize the mechanical stress

Scanning this QR code will take you to a video for the article.

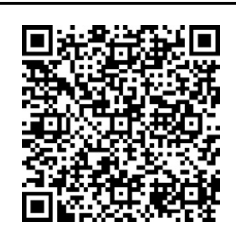




\section{Abbreviations and Acronyms \\ AVR = aortic valve replacement \\ $\mathrm{CPB}=$ cardiopulmonary bypass \\ $\mathrm{EOA}=$ effective orifice area \\ IE $=$ infective (prosthetic) endocarditis \\ iEOA $=$ indexed effective orifice area \\ NSVD $=$ nonstructural valve dysfunction \\ PPM = patient-prosthesis mismatch \\ SVD = structural valve deterioration}

to the leaflets. The Trifecta valve has demonstrated good hemodynamic performance at rest, ${ }^{2-4}$ during effort, ${ }^{5}$ and at the 3-year follow-up. ${ }^{6}$ This characteristic makes it very attractive, especially for patients with a small aortic annulus. In addition, the Trifecta Durability Study has reported encouraging results over a median 0.9 year of follow-up for 1014 patients (844 patient-years), with a freedom from valve explant of $99.4 \%$ at 2 years. ${ }^{7}$ In the same study, there was only 1 case of explant due to structural valve deterioration (SVD), suggesting good durability of this device in the initial follow-up.

Nonetheless, longer-term follow-up is of paramount importance to understand the performance of this valve device and guide the choice of prosthesis in clinical practice. In the present investigation, the primary purpose was to evaluate our single-center experience and follow-up (average, 2.2 years; 1647.8 patient-years, up to 6.9 years) with 824 Trifecta implants for AVR. Our secondary objective was to evaluate the immediate postoperative hemodynamic performance of the Trifecta valve in the entire study cohort $(n=824)$.

\section{PATIENTS AND METHODS}

Our center began implanting the Trifecta valve in 2008, and participated in the Trifecta Durability Study. In January 2015, we retrospectively reviewed the electronic records of patients who received AVR using this bioprosthesis until December 31, 2014. The performance of any concomitant procedure at the time of AVR and a history of previous cardiac surgery were not exclusion criteria. Preoperative, intraoperative, and early postoperative data were collected prospectively in an electronic database at the time of patient discharge, as described previously. ${ }^{8}$ The database includes all patients receiving cardiac surgery at our institution; it is managed by research nurses and regularly checked for completion and consistency under the supervision of the surgical team. For the purpose of the present study, a retrospective clinical follow-up was performed between January and May 31, 2015. Practitioners (referring cardiologists and general medicine doctors) were provided with questionnaires that enquired about the patient's vital status, occurrence and timing of any adverse events (both valve-related and non-valve-related), and functional status. In cases of incomplete or missing information, the patient or family was contacted; local governmental authorities were asked to confirm the patient's vital status in case of missing data.

Valve-related adverse events were defined according to current guidelines $^{9}$ and included SVD (changes intrinsic to the device causing dysfunction, evident at echocardiography, reoperation, or autopsy), nonstructural valve dysfunction (NSVD; any abnormality not intrinsic to the valve itself resulting in stenosis, regurgitation, or hemolysis, evident at echocardiography, reoperation, or autopsy) and operated valve infective endocarditis (IE). Valve thrombosis and embolic events (both cerebral and noncerebral) were aggregated as thromboembolic complications. Hemorrhagic complications were reported in the overall population and stratified according to the administration of anticoagulant therapy. Reinterventions were both reported in aggregate form and stratified according to type (either open reoperative surgery or interventional catheter-based procedures). Valve-related mortality was death related to any of the above adverse events or to reoperation on the index valve. Causes of death were determined by review of hospital records and instrumental data. All complications and fatal events for which no demonstrated nonvalvular cause was known (including sudden death) were considered valve-related events. Mortality resulting from cerebrovascular events during follow-up was also assumed to be valve-related.

Regarding early complications, we distinguished among perioperative stroke (defined as new focal neurologic deficit or coma evident immediately after resolution of anesthesia), which was considered non-valve-related, and postoperative stroke (defined as new focal neurologic deficit or coma that became evident after a normal awakening from anesthesia), which was considered valve-related unless demonstrated otherwise. This "conservative" approach was followed in compliance with guidelines on data reporting, and should be kept in mind while interpreting the results. Adverse events were categorized as either early (when occurring during the hospitalization during which the operation was performed, even if after 30 days and including patients transferred to other acute care facilities, or when occurring after discharge from the hospital but before the end of the 30th postoperative day) or late in all other cases.

Because all patient data were managed anonymously, and because the study did not entail any modification to standard treatment protocols, the need for patient informed consent for inclusion was waived. The study database was declared to the online database of the Commission Nationale de l'Informatique et des Libertès [National Committee for Informatics and Freedom] under dossier number 1207754, in accordance with French law.

\section{Surgical Technique}

For all cases included in the present study, the Trifecta bioprostheses were implanted through a full median sternotomy and mildly hypothermic cardiopulmonary bypass (CPB). Myocardial protection was achieved through cold crystalloid cardioplegia or isothermic hyperkalemic blood cardioplegia. Valves were implanted with a supra-annular technique, using interrupted, noneverting, U-shaped stitches. Video 1 shows the implantation of a Trifecta GT device (an improved model of the Trifecta valve now in clinical use). Particular care must be devoted to valve sizing even at the supra-annular level (to avoid valve deformation within narrow sinotubular junction anatomies) and to aortotomy suturing to avoid leaflet impingement into sutures. Particular features of the Trifecta GT device with respect to the previous model include an improved sewing ring (facilitating suture passing and sliding as well as valve seating into the annulus) and a modified valve holder (to facilitate handling without risk of stent deformation during parachuting). It is also characterized by increased stent radiopacity to aid a valve-in-valve procedure in the event of SVD.

\section{Postoperative Management}

All patients underwent transthoracic echocardiography before discharge. At this time, data on valve function, including peak and mean transvalvular gradient, degree and topography of regurgitation, left ventricular ejection fraction, crude effective orifice area (EOA), and indexed effective orifice area (iEOA), were collected prospectively. A team of experienced in-house cardiologists performed echocardiography, and data were recorded prospectively as part of our electronic database. 


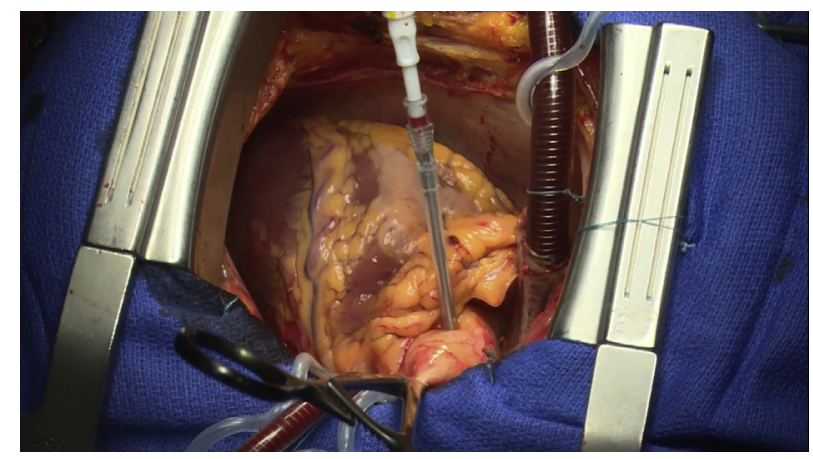

VIDEO 1. Implantation of a 19-mm Trifecta GT valve through full sternotomy for severe aortic stenosis. After aortic cross-clamping and injection of antegrade cardioplegia, an oblique aortotomy is performed, and the native valve is excised. This case is characterized by small annular diameter; both intra-annular and supra-annular sizing are performed to anticipate the overall device fit within the aortic root. A supra-annular technique through interrupted U-shaped stitches is used. Sutures are passed through the prosthetic sewing ring, which features markers at the bottom of each stent post. The sewing ring is designed to facilitate suture gliding when the valve is parachuted, and is shaped to conform to the 3-dimensional anatomy of the native annulus. The valve holder is modified to push at the midpoints between stent posts and avoid the risk of stent deformation during parachuting. Only 1 suture must be divided to liberate the valve. After knot tying, the aortotomy is sutured, taking care to avoid leaflet impingement into the sutures. Video available at: http://www.jtcvsonline.org/article/S0022-5223(16)31067-4/addons.

Patient-prosthesis mismatch (PPM) was classified as severe (iEOA $\leq 0.65 \mathrm{~cm}^{2} / \mathrm{m}^{2}$ ), moderate (iEOA $\leq 0.85 \mathrm{~cm}^{2} / \mathrm{m}^{2}$ ), or absent (iEOA $\left.>0.85 \mathrm{~cm}^{2} / \mathrm{m}^{2}\right) .{ }^{10}$ Given the relatively few number of patients receiving $27-\mathrm{mm}$ and $29-\mathrm{mm}$ valves, hemodynamic data for these 2 sizes were aggregated. No modifications were made to our postoperative management protocol during the study period. Patients received no oral anticoagulant medications after surgery (unless indicated otherwise), and were given oral acetylsalicylate $160 \mathrm{mg}$ /day. Antiaggregant therapy was maintained after discharge. In the immediate postoperative period, antithrombotic prophylaxis was provided through subcutaneous administration of low molecular weight heparin (4000 IU/day) until mobilization.

\section{Endpoints}

We evaluated the early postoperative clinical findings (valve-related and non-valve-related events) after implantation of the Trifecta valve for AVR. In follow-up analysis, we addressed midterm overall survival, event-free survival, SVD-free survival, and reoperation-free survival after implantation of the Trifecta valve for AVR. Finally, we evaluated the early postoperative hemodynamic performance of the Trifecta valve in the entire study cohort.

\section{Statistical Analysis}

Statistical analysis was performed using SAS version 9.33 (SAS Institute, Cary, NC). Continuous data are presented as mean \pm standard deviation; categorical date, as number and percentage. The normality of data distribution was evaluated using the Kolmogorov-Smirnoff test. Intergroup comparison was performed for the average CPB and aortic clamp times in isolated AVR and nonisolated AVR subgroups. A normal distribution of these variables was confirmed; thus, intergroup comparison was done using the Student's $t$ test (continuous variables). All tests were 2-tailed, and the $\alpha$ level was set at 0.05 . A multiple logistic regression model (stepwise selection method) was built to identify baseline factors associated with operative mortality. Follow-up analysis was performed using KaplanMeier (actuarial) methodology, and the results are graphically presented as curves of mortality risk. Survival rates are reported. Secondarily, stratified survival analysis at follow-up was performed according to the presence or absence of severe/moderate PPM after surgery.

\section{RESULTS \\ Early Results}

During the study period, 824 patients underwent AVR using a Trifecta valve and were included in our analysis; their baseline demographic data are summarized in Table 1. Predominant stenosis was the most frequent type of native valve dysfunction; in fact, patients presenting with degenerative calcified aortic stenosis composed the majority of our study population. The rate of baseline comorbidities was limited, which is reflected by the relatively low average value of logistic EuroSCORE I. There were few nonelective cases $(1.6 \%)$, and the rate of concomitant procedures was $7.3 \%$. Perioperative characteristics are reported in Table 2. Average $\mathrm{CPB}$ and aortic cross-clamp times were significantly greater in patients in whom a concomitant procedure had been performed $(P<.001$ for both). Conversely, average CPB and aortic clamp time were limited $(48.1 \pm 14.9$ minutes and $38 \pm 11.3$ minutes, respectively) in patients with isolated AVR. Figure 1 reports the size distribution of the implanted valves. A $23-\mathrm{mm}$ valve was most frequently used $(36.4 \%)$, followed by a $21-\mathrm{mm}$ valve $(29.2 \%)$.

Overall operative mortality was $3.8 \%(\mathrm{n}=31)$, including $2.7 \%$ in patients undergoing isolated AVR and $8.1 \%$ in those undergoing combined surgery $(P=.0013)$. Causes

TABLE 1. Demographic data

\begin{tabular}{lc}
\hline \multicolumn{1}{c}{ Characteristic } & Value \\
\hline Age, $\mathrm{y}$, mean $\pm \mathrm{SD}$ & $75.4 \pm 7.7$ \\
Male sex, $\mathrm{n}(\%)$ & $461(55.9)$ \\
New York Heart Association class III or IV, $\mathrm{n}(\%)$ & $276(33.5)$ \\
Extracardiac arteriopathy, $\mathrm{n}(\%)$ & $146(17.7)$ \\
Renal insufficiency, $\mathrm{n}(\%)$ & $38(4.6)$ \\
Chronic obstructive pulmonary disease, $\mathrm{n}(\%)$ & $106(12.9)$ \\
Previous stroke, $\mathrm{n}(\%)$ & $36(4.4)$ \\
Hypertension, $\mathrm{n}(\%)$ & $580(70.4)$ \\
Tobacco, $\mathrm{n}(\%)$ & $162(19.7)$ \\
Diabetes, $\mathrm{n}(\%)$ & $137(16.6)$ \\
Hematologic disorder, $\mathrm{n}(\%)$ & $27(3.3)$ \\
Hepatic insufficiency, $\mathrm{n}(\%)$ & $6(0.7)$ \\
Neoplasm, $\mathrm{n}(\%)$ & $76(9.2)$ \\
Left ventricular ejection fraction, $\%$, mean $\pm \mathrm{SD}$ & $60.8 \pm 10.9$ \\
Native valve dysfunction, $\mathrm{n}(\%)$ & \\
$\quad$ Predominant stenosis & $791(96)$ \\
Predominant regurgitation & $33(4)$ \\
Bicuspid aortic valve, $\mathrm{n}(\%)$ & $79(9.6)$ \\
Infective endocarditis, $\mathrm{n}(\%)$ & $6(0.7)$ \\
Previous cardiac operation, $\mathrm{n}(\%)$ & $11(1.3)$ \\
Logistic EuroSCORE I, \%, mean $\pm \mathrm{SD}$ & $8.7 \pm 5.6$ \\
\hline
\end{tabular}

$S D$, Standard deviation. 
TABLE 2. Perioperative characteristics

\begin{tabular}{lc}
\hline \multicolumn{1}{c}{ Characteristic } & Value \\
\hline Nonelective priority, $\mathrm{n}(\%)$ & $13(1.6)$ \\
Associated procedures, $\mathrm{n}(\%)$ & \\
$\quad$ Other valve surgery & $11(1.3)$ \\
Other valve surgery and coronary bypass & $1(0.1)$ \\
Thoracic aortic surgery & $30(3.6)$ \\
Thoracic aortic surgery and coronary bypass & $2(0.2)$ \\
Coronary bypass & $2(0.2)$ \\
Miscellaneous procedures & $14(1.7)$ \\
Cardiopulmonary bypass time, min, mean $\pm \mathrm{SD}$ & \\
Overall population & $56 \pm 24.6$ \\
Isolated AVR & $48.1 \pm 14.9 *$ \\
Associated procedure & $88.6 \pm 29.6 *$ \\
Cross-clamp time, min, mean $\pm \mathrm{SD}$ & \\
Overall population & $44.6 \pm 19.7$ \\
Isolated AVR & $38 \pm 11.3 \dagger$ \\
Associated procedure & $71.6 \pm 23.7 \dagger$ \\
\hline$S D$, Standard deviation; $A V R$, aortic valve replacement. $* P<.001 . \dagger P<.001$.
\end{tabular}

of operative mortality were extracardiac in 15 patients $(1.8 \%)$ and cardiac nonvalvular in 16 patients $(1.9 \%)$. No operative deaths were attributed to valvular causes. The early postoperative (30 days) complications are described in Table 3. There were 9 valve-related early complications $(1.1 \%)$, including 4 cerebral strokes (of which only 1 caused permanent damage), 1 case of early IE (requiring reoperation), and 4 cases of NSVD. Among the NSVD cases, 2 demonstrated severe transvalvular regurgitation on echocardiography; reoperation identified engagement of the noncoronary leaflet of the bioprosthesis into the suture of the oblique aortotomy. At reoperation, these 2 cases were managed through section of the suture without the need for valve re-replacement. The 2 bioprostheses were functioning normally at the last available follow-up. One other early NSVD case involved severe transvalvular regurgitation evident on echocardiography; at reoperation, valve oversizing (a 27-mm valve) was found in this patient, who had a relatively narrow sinotubular junction, which

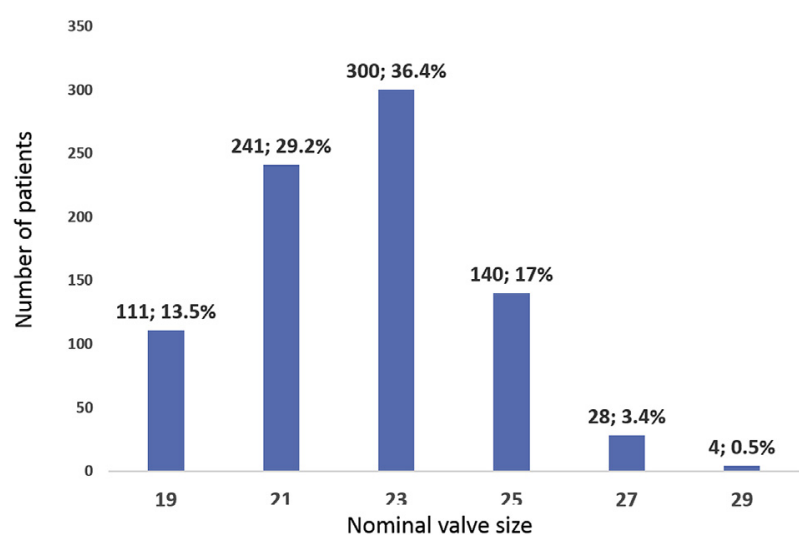

FIGURE 1. Distribution of nominal valve sizes. The 23-mm diameter valve was most frequently implanted.
TABLE 3. Early postoperative morbidity (within 30 days of surgery)

\begin{tabular}{lc}
\multicolumn{1}{c}{ Characteristic } & n (\%) \\
\hline Valve-related complications & \\
Stroke & $4(0.5)$ \\
NSVD & $4(0.5)$ \\
IE & $1(0.1)$ \\
Reoperation & $5(0.6)$ \\
Noncerebral embolism & 0 \\
Early reoperation (NSVD, IE, non-valve-related) & $9(1.1)$ \\
Non-valve-related complications & \\
Revision for bleeding & $21(2.5)$ \\
Prolonged ventilation & $33(4)$ \\
Renal failure (with dialysis) & $13(1.6)$ \\
Renal failure (without dialysis) & $37(4.5)$ \\
Reoperation & $4(0.5)$ \\
Atrial fibrillation & $361(44)$ \\
Pacemaker implantation & $16(1.9)$ \\
\hline NSVD, Nonstructural valve dysfunction; $I E$, infective (prosthetic) endocarditis.
\end{tabular}

resulted in deformation of the upper valve stent and consequent leaflet malcoaptation. The patient was treated by replacing the oversized valve with a new $23-\mathrm{mm}$ Trifecta valve. The fourth case of NSVD was characterized by elevated transvalvular gradients at follow-up echocardiography in a patient who had undergone AVR with a 19-mm Trifecta valve plus septal myectomy for aortic stenosis and obstructive cardiomyopathy. At reoperation, persistent subvalvular stenosis and valve oversizing were noted, and the patient was treated by iterative septal myectomy and AVR with a 17-mm mechanical valve prosthesis. There were no early SVD cases. We observed 4 additional reoperations that were not valve-related. The rate of early valve-related reoperation was $0.6 \%$ (with no cases of associated mortality); the average interval after primary surgery was $7.6 \pm 6.9$ days. Multiple logistic regression identified advanced age, renal insufficiency, coexisting neoplasm, and left heart failure as significant predictors of operative mortality.

Table 4 reports the hemodynamic findings obtained by transthoracic echocardiography immediately before discharge. The mean average transvalvular gradient was $<10 \mathrm{~mm} \mathrm{Hg}$ in all valve size subgroups except the 19-mm subgroup. Severe PPM was observed in the 19-mm, 21-mm, and 23-mm subgroups, albeit at extremely low rates $(5 \%, 1 \%$, and $1 \%$, respectively).

\section{Follow-up Results}

We obtained data for all of the 793 patients who were discharged alive from the hospital and entered follow-up ( $100 \%$ follow-up completion). The average duration of follow-up was $2.2 \pm 1.3$ years (range, 0.03-6.9 years; median, 2 years); a total of 1747.6 patient-years were available for analysis (Figure 2). During the follow-up, 54 late deaths occurred ( $6.8 \%$ of the patients who were discharged alive), 
TABLE 4. Valve hemodynamics at discharge stratified according to valve size

\begin{tabular}{lccccr}
\hline & \multicolumn{5}{c}{ Valve size } \\
\cline { 2 - 6 } \multicolumn{1}{c}{ Characteristic } & $\mathbf{1 9}$ & $\mathbf{2 1}$ & $\mathbf{2 3}$ & $\mathbf{2 5}$ & $\mathbf{2 7 - 2 9}$ \\
\hline $\begin{array}{l}\text { Average transvalvular } \\
\quad \text { gradient, mm Hg }\end{array}$ & 13 & 9.8 & 9.4 & 7.4 & 6.4 \\
$\begin{array}{l}\text { Peak transvalvular } \\
\text { gradient, mm Hg }\end{array}$ & 23.9 & 18.5 & 18.2 & 14.3 & 12.7 \\
$\begin{array}{l}\text { EOA, cm } \\
\text { iEOA, cm }{ }^{2} / \mathrm{m}^{2}\end{array}$ & 1.5 & 1.8 & 2.1 & 2.4 & 2.8 \\
$\begin{array}{l}\text { PPM, \% } \\
\quad \text { Severe }\end{array}$ & 0.9 & 1.1 & 1.1 & 1.3 & 1.5 \\
$\quad \begin{array}{l}\text { Moderate } \\
\quad \text { None }\end{array}$ & $53 \%$ & $1 \%$ & $1 \%$ & 0 & 0 \\
\hline
\end{tabular}

$E O A$, Crude effective orifice area; $i E O A$, indexed effective orifice area; $P P M$, patientprosthesis mismatch.

at an average of $1.8 \pm 1.3$ years after surgery; thus, 739 patients were alive at the end of follow-up $(93.2 \%$ of the patients who were discharged alive). Causes of late death were extracardiac in 33 cases $(61.1 \%$ of late deaths), cardiac nonvalvular in 12 cases $(22.2 \%)$, and valverelated in 9 cases $(16.7 \%$, including 7 deaths of unknown cause and 2 deaths due to prosthetic valve IE). At the 5 -year follow-up, the overall survival in the entire study population was $74.9 \pm 8.5 \%$, and the freedom from valve-related death was $97.8 \pm 0.9 \%$. Figure $3, A$ and $B$, depicts the risk of death due to any cause and due to valve-related causes.

During follow-up, 6 SVD events occurred, at an average of $3.4 \pm 1.6$ years after valve implantation. The 5-year Kaplan-Meier SVD-free survival rate was $98 \% \pm 0.9 \%$. Figure 4, A, depicts the risk of death or SVD. Among these 6 SVD events, 5 required reintervention and 1 was managed

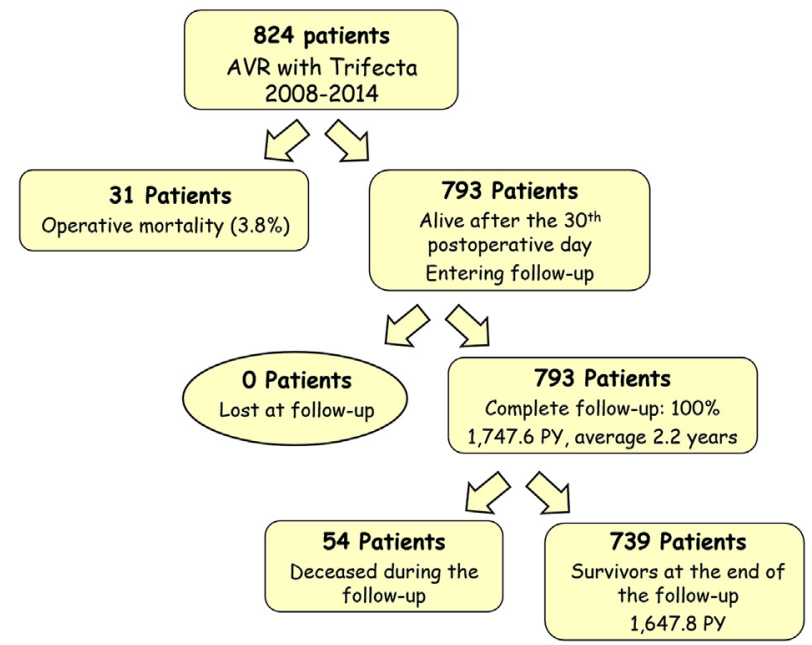

FIGURE 2. Study workflow. Among the 824 patients initially included, $739(89.7 \%)$ were alive at the end of the follow-up. A total of 1747.6 patient/years $(P / Y)$ were available. AVR, Aortic valve replacement. by medical treatment only (case 5). The freedom from reintervention (any type) for SVD was $98 \% \pm 0.9$ at 5 years. Figure $4, B$, depicts the risk of death or any reintervention for SVD, including a valve-in-valve procedure. Reinterventions were open redo surgery in 3 cases (cases 3, 4, and 6), and transcatheter valve-in-valve procedure in 2 cases (cases 1 and 2). The 5-year freedom from open redo surgery for SVD was $98.9 \% \pm 0.6 \%$ at 5 years. Figure $4, C$, depicts the risk of death or open redo surgery for SVD. Modalities of SVD were severe intravalvular regurgitation presenting with rapid onset of heart failure in 4 cases (cases 1-4), moderate intravalvular regurgitation presenting with progressive dyspnea in 1 case (case 5), and severe valve stenosis presenting with worsening dyspnea in 1 case (case 6). At reoperation, a noncalcified leaflet tear with leaflet disinsertion from a stent post was discovered in cases 3 and 4, and diffuse tissue thickening and calcification without tears were identified in case 6 . Abstinence from reintervention in case 5 , an elderly patient with reduced physical activity owing to several comorbidities, was chosen based on the nonsevere degree of valve dysfunction, moderate symptoms, and the risks associated with both redo surgery and transcatheter therapy in this patient.

Interestingly, patients with severe or moderate PPM detected immediately after surgery did not have significantly different overall survival at follow-up compared with patients without PPM $(P=.18, \log$-rank test). Similar findings were obtained for freedom from valve-related death and freedom from SVD $(P=.93$ and .50 , respectively). There were 12 cases of late IE (5-year actuarial freedom, $97.7 \% \pm 0.7 \%$ ). Reoperation for IE was required in 6 of these case, whereas the other 6 were managed with medical therapy alone. Only 1 case of NSVD was identified during follow-up, which did not require reintervention (5-year freedom, $99.8 \% \pm 0.2 \%$ ). There were 7 hemorrhagic events, 6 of which occurred in patients receiving oral anticoagulants for chronic atrial fibrillation (5-year freedom, $98.6 \% \pm 0.5 \%$ ). There were no thromboembolic events during the available follow-up. Globally, the 5-year actuarial freedom from any valve-related reintervention was $96.9 \% \pm 1 \%$. Figure 3 , $C$, depicts the risk of death or any valve-related reintervention. Table E1 summarizes the adverse events observed at follow-up.

\section{DISCUSSION}

The St. Jude Trifecta valve has been used in clinical practice since 2007. As a typical feature, the bovine pericardial tissue is mounted outside the stent frame. This translates to optimized EOA and excellent hemodynamic properties. A considerable body of independent literature beyond the sponsored Trifecta Durability Study has consistently demonstrated low average transvalvular gradients and very low rates of PPM in the immediate postoperative 

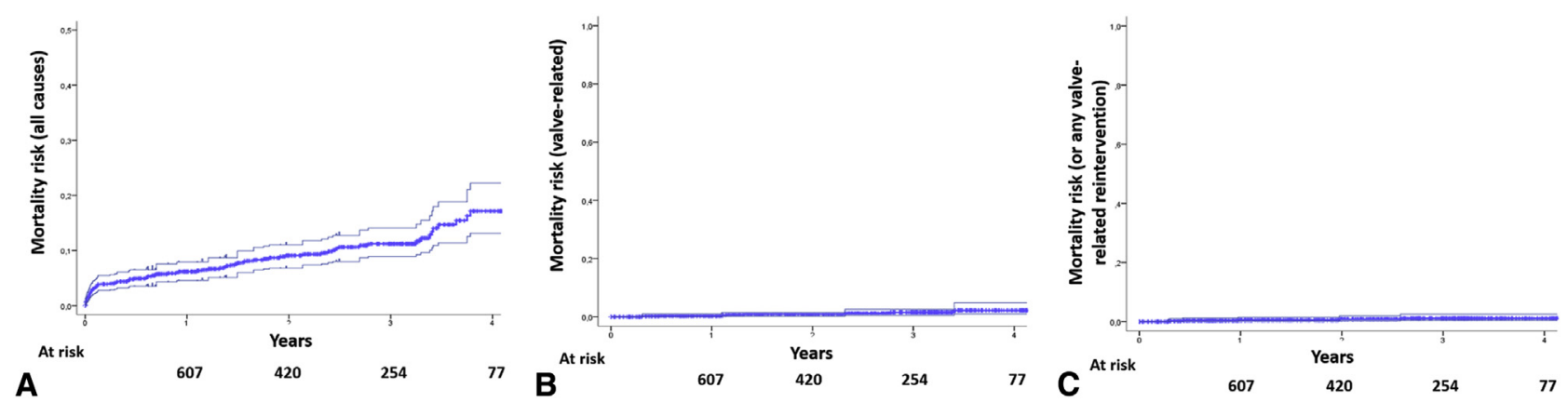

FIGURE 3. Kaplan-Meier curves of mortality risk in the entire study population. Confidence limits are indicated as areas around the curves. A, Risk of death due to any cause. B, Risk of valve-related death. C, Risk of any valve-related reintervention.

period compared with other valve devices. ${ }^{2,3,11-13}$ Although the impact of PPM on long-term patient outcomes is a matter of debate, ${ }^{14,15}$ the Trifecta valve offers specific advantages in patients with a small aortic annulus and patients at high risk for postoperative PPM. Recently, our group reported 3 years of data on the hemodynamic performance of this device, which suggests that its hemodynamic profile is preserved at the midterm followup. ${ }^{6}$ The population analyzed in that study is included in our present cohort. Although 3-year hemodynamic results are not available for the overall cohort presented here, we have confirmed the excellent hemodynamic profile of the Trifecta valve at the time of hospital discharge (Table 4).

The main aim of the present study was to evaluate the durability of the innovative Trifecta valve. For this purpose, we analyzed the largest single-center population reported to date, with the longest available follow-up $(n=824 ; 1747.6$ patient-years; average follow-up, 2.2 years). Follow-up was facilitated by the propensity of the patients treated at our center to remain in the region after surgery, which accounts for the $100 \%$ rate of follow-up completion. Durability is a major requirement for modern bioprostheses, given the growing trend toward their use in younger individuals. ${ }^{16}$ This feature is even more important in the current era, when the results of surgical AVR are considered the benchmark reference compared with transcatheter valve therapy.
In our study population, a total of 6 SVD events occurred at an average of $3.4 \pm 1.6$ years after valve implantation $(98 \%$ $\pm 0.9 \%$ Kaplan-Meier freedom from SVD at 5 years). This rate of freedom from SVD is slightly lower than that documented for other bioprostheses in a previously published large series, which reported a 5-year freedom from SVD values of $99.3 \%$ (Kaplan-Meier) and 99.4\% (competingrisks methodology) for a third-generation porcine valve in the aortic position. ${ }^{8}$ In a smaller previous series, the 5-year freedom from SVD for a third-generation pericardial valve was $100 \%,{ }^{16}$ although that study was potentially limited by sample bias. In more recent investigations, the 5-year actuarial freedom from SVD values were 99.8\% for a third-generation pericardial valve ${ }^{17}$ and $99.2 \%$ (Kaplan-Meier) and 99.3\% (competing-risks) for a second-generation porcine valve. ${ }^{18}$ One previous series that focused on the Trifecta valve reported no cases of reoperation for SVD at 2 years, but the rate of non-reoperation for SVD was not reported. ${ }^{11}$ Although the number of SVD events reported in our series is limited and may be subject to population bias, the occurrence of these events underscores the need for continued and exhaustive follow-up of the implanted patients.

Calcified SVD occurred in only 1 case in our series. The Linx anticalcification treatment has been reported to remove up to $94 \%$ of phospholipids from leaflet tissue
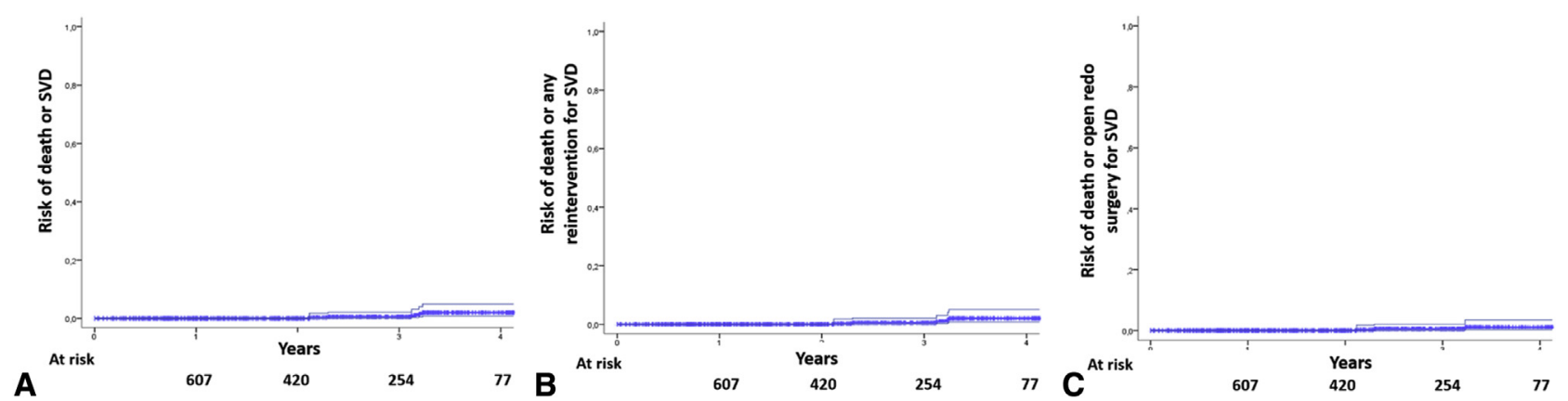

FIGURE 4. Kaplan-Meier curves of mortality risk in the entire study population. Confidence limits are indicated as areas around the curves. A, Risk of death or SVD. B, Risk of death or any reintervention for SVD (including transcatheter valve-in-valve). C, Risk of death or open redo surgery for SVD. 
and has demonstrated clinical effectiveness at midterm follow-up in porcine bioprostheses. ${ }^{1,19}$ On the other hand, 5 out of our 6 SVD cases presented with noncalcified leaflet tears, extended over the height of one stent post (4 cases) or at the bottom of one leaflet (1 case) without macroscopically overt calcification. This type of failure is rather specific with respect to other valve devices. The clinical presentation was also characteristic, involving a sudden onset of severe dyspnea and heart failure. Conversely, calcified SVD is traditionally considered to be slowly progressive, with a sometimes indolent clinical course. Physicians involved in the follow-up of these patients should consider the possibility of rapid onset of clinical manifestations of Trifecta SVD. Interestingly, the Trifecta Durability Study (median follow-up, 0.9 years) reported 1 case of SVD requiring valve explantation but with no significant leaflet calcification. Few similar cases have been reported in the literature, ${ }^{20}$ and only 1 additional case of early calcific SVD without tear has been reported. ${ }^{21} \mathrm{An}$ in-depth investigation of valve design and manufacturing is needed to clarify the underlying mechanism for this phenomenon.

The impact of PPM on patient outcomes is a matter of debate. In the present series, severe/moderate PPM was not associated with an increased risk of death from any cause, of valve-related death, or of SVD at follow-up. The interpretation of PPM and its significance are beyond the scope of the present work. Nonetheless, we believe that the expected benefits from avoiding PPM through annular enlargement should be balanced against the associated increased risk of operative mortality and morbidity.

Our present study also highlights the need for special precautions when sizing and implanting the Trifecta bioprosthesis. Two early NSVD cases were related to valve oversizing. Because the Trifecta titanium stent is deformable mainly in its upper portion, a narrow sinotubular junction anatomy may lead to leaflet malcoaptation from stent deformation. Moreover, oversizing may explain a case of late fusion of a valve leaflet into the aortic wall reported in the literature. ${ }^{20}$ The specific valve design carries a risk of noncoronary leaflet impingement in sutures, especially if the aortotomy is extended into the noncoronary sinus and supplementary hemostatic stitches are added after release of the aortic cross-clamp. Particular attention must be given to precise suturing of the aortotomy. Coronary obstruction is another potential complication that may be related to valve oversizing.

The specific features of the Trifecta valve (namely the outer position of the pericardial sheet and its globally rectangular shape) have been associated with an increased risk of coronary obstruction during transcatheter valve-invalve procedures. ${ }^{22}$ Two patients in our present series considered to be at high risk for reoperation were successfully treated with a valve-in-valve procedure without developing coronary obstruction. Accurate preprocedural imaging assessment and proper choice of the transcatheter device, as well as precise deployment, are crucial. ${ }^{23}$ Other reports have confirmed the feasibility of this approach. ${ }^{24}$ According to recommendations, ${ }^{9}$ freedom from open redo surgery and from transcatheter reintervention on the index valve was presented here both in aggregate form and separately.

Similarly, we emphasize the importance of defining SVD as the occurrence of intrinsic valve changes, irrespective of the performance of any reintervention, to avoid any underestimation of the SVD rate. ${ }^{25}$ In contrast to previous work, here we did not present results according to the competing-risks methodology, given the limited number of SVD events and the relatively short average duration of follow-up. The competing-risks (or "actual") method is recognized as a key tool for data interpretation in long-term valve durability studies. ${ }^{26-28}$ For the same reason, we did not stratify the results according to patient age at surgery.

Limitations of the present study include the impossibility of providing EuroSCORE II data, and the lack of complete echocardiographic follow-up at a single center. Future studies about valve durability will need to analyze separately the results obtained with the first model of Trifecta valve (which is the object of the present paper) and those achieved through its new model (Trifecta GT). Video 1 shows the implantation of a 19-mm Trifecta GT valve. This improved version of the Trifecta is available in Europe since the second quarter of 2016, and is characterized by facilitated suture gliding through the sewing ring and seating into the native annulus, a modified holder to facilitate parachuting and seating, and increased stent radiopacity. The hemodynamic properties are comparable to those observed for the previous version. This device is expected to the object of future dedicated investigations.

In conclusion, here we have evaluated the durability of the Trifecta bioprosthesis for AVR in the largest published single-center cohort with the longest duration of followup reported to date. Our data confirm the excellent immediate hemodynamic properties of this device. Accurate sizing and proper implantation technique are vitally important. Durability is consistent, although rigorous follow-up is required in future trials, given the characteristics and the timing of the few SVD events observed herein. Such SVD events may be linked with the design of this prosthesis and its mechanical behavior. Improvements in this area may be achieved by recent improvements in the device. Practitioners need to be aware of the potential modalities of failure and of their clinical presentation, and further dedicated studies are needed to explore their pathophysiology.

\section{Conflict of Interest Statement}

Authors have nothing to disclose with regard to commercial support. 


\section{References}

1. Jamieson WR, Lewis CT, Sakwa MP, Cooley DA, Kshettry VR, Jones KW, et al St Jude Medical Epic porcine bioprosthesis: results of the regulatory evaluation. J Thorac Cardiovasc Surg. 2011;141:1449-54.e2.

2. Remadi JP, Levy F, Szymanski C, Nzomvuama A, Zogheib E, Gun M, et al. Early hemodynamics results of aortic valve replacement with the new St Jude Trifecta bioprosthesis. Int J Cardiol. 2014;174:755-7.

3. Permanyer E, Estigarribia AJ, Ysasi A, Herrero E, Semper O, Llorens R. St. Jude Medical Trifecta $^{\mathrm{TM}}$ aortic valve perioperative performance in 200 patients. Interact Cardiovasc Thorac Surg. 2013;17:669-72.

4. Yadlapati A, Diep J, Barnes M, Grogan T, Bethencourt DM, Vorobiof G. Comprehensive hemodynamic comparison and frequency of patient-prosthesis mismatch between the St. Jude Medical Trifecta and Epic Bioprosthetic aortic valves. J Am Soc Echocardiogr. 2014;27:581-9.

5. Levy F, Donal E, Bière L, Szymanski C, Remadi JP, Flécher E, et al. Hemodynamic performance during exercise of the new St. Jude Trifecta aortic bioprosthesis: results from a French multicenter study. J Am Soc Echocardiogr. 2014;27:590-7.

6. Ruggieri VG, Anselmi A, Chabanne C, Lelong B, Flecher E, Verhoye JP, et al Three-year haemodynamic performance of the St Jude Trifecta bioprosthesis. Eur J Cardiothorac Surg. 2016;49:972-7.

7. Bavaria JE, Desai ND, Cheung A, Petracek MR, Groh MA, Borger MA, et al The St Jude Medical Trifecta aortic pericardial valve: results from a global, multicenter, prospective clinical study. J Thorac Cardiovasc Surg. 2014;147:590-7.

8. Anselmi A, Flécher E, Ruggieri VG, Harmouche M, Langanay T, Corbineau H, et al. Long-term results of the Medtronic Mosaic porcine bioprosthesis in the aortic position. J Thorac Cardiovasc Surg. 2014;147:1884-91.

9. Akins CW, Miller DC, Turina MI, Kouchoukos NT, Blackstone EH, Grunkemeier GL, et al. Guidelines for reporting mortality and morbidity after cardiac valve interventions. J Thorac Cardiovasc Surg. 2008;135:732-8.

10. Blais C, Dumesnil JG, Baillot R, Simard S, Doyle D, Pibarot P. Impact of valve prosthesis-patient mismatch on short-term mortality after aortic valve replacement. Circulation. 2003;108:983-8.

11. Modi A, Budra M, Miskolczi S, Velissaris T, Kaarne M, Barlow CW, et al. Hemodynamic performance of Trifecta: single-center experience of 400 patients. Asian Cardiovasc Thorac Ann. 2015;23:140-5.

12. Wendt D, Thielmann M, Plicht B, Aßmann J, Price V, Neuhäuser M, et al. The new St Jude Trifecta versus Carpentier-Edwards Perimount Magna and Magna Ease aortic bioprosthesis: is there a hemodynamic superiority? J Thorac Cardiovasc Surg. 2014; 147:1553-60.

13. Dell'Aquila AM, Schlarb D, Schneider SR, Sindermann JR, Hoffmeier A, Kaleschke G, et al. Clinical and echocardiographic outcomes after implantation of the Trifecta aortic bioprosthesis: an initial single-centre experience. Interact Cardiovasc Thorac Surg. 2013;16:112-5.

14. Tully PJ, Aty W, Rice GD, Bennetts JS, Knight JL, Baker RA. Aortic valve prosthesis-patient mismatch and long-term outcomes: 19-year single-center experience. Ann Thorac Surg. 2013;96:844-50.
15. Pibarot P, Dumesnil JG. Prosthesis-patient mismatch: definition, clinical impact, and prevention. Heart. 2006;92:1022-9.

16. McClure RS, McGurk S, Cevasco M, Maloney A, Gosev I, Wiegerinck EM, et al Late outcomes comparison of nonelderly patients with stented bioprosthetic and mechanical valves in the aortic position: a propensity-matched analysis. J Thorac Cardiovasc Surg. 2014:148:1931-9.

17. McClure RS, Narayanasamy N, Wiegerinck E, Lipsitz S, Maloney A, Byrne JG et al. Late outcomes for aortic valve replacement with the Carpentier-Edward pericardial bioprosthesis: up to 17-year follow-up in 1,000 patients. Ann Thorac Surg. 2010;89:1410-6.

18. Ruggieri VG, Flecher E, Anselmi A, Lelong B, Corbineau H, Verhoye JP, et al Long-term results of the Carpentier-Edwards supraannular aortic valve prosthesis. Ann Thorac Surg. 2012;94:1191-7.

19. Lehmann S, Walther T, Leontjev S, Kempfert J, Rastan A, Garbade J, et al. Mid term results after Epic xenograft implantation for aortic, mitral, and double valve replacement. J Heart Valve Dis. 2007;16:641-8

20. Campisi S, Camilleri L, Innorta A, Azarnoush K. Early failures of Trifecta aortic bioprosthesis. J Thorac Cardiovasc Surg. 2014;148:e133-4.

21. Saxena P, Greason KL, Schaff HV. Early structural valve deterioration of the Trifecta aortic valve biological prosthesis: a word of caution. J Thorac Cardio vasc Surg. 2014;147:e10-1.

22. Dvir D, Webb J, Brecker S, Bleiziffer S, Hildick-Smith D, Colombo A, et al Transcatheter aortic valve replacement for degenerative bioprosthetic surgical valves: results from the global valve-in-valve registry. Circulation. 2012;126: 2335-44.

23. Verhoye JP, Harmouche M, Soulami RB, Thebault C, Boulmier D, Leguerrier A, et al. Feasibility of valve-in-valve procedure for degenerated St Jude Medical Trifecta bioprosthesis. J Heart Valve Dis. 2015;24:484-6.

24. Haussig S, Schuler G, Linke A. Treatment of a failing St. Jude Medical Trifecta by Medtronic Corevalve Evolut valve-in-valve implantation. JACC Cardiovasc Interv. 2014;7:e81-2.

25. Jamieson WR. Bioprosthetic durability assessment: importance of complete data J Thorac Cardiovasc Surg. 2011;141:596.

26. Grunkemeier GL, Jin R, Eijkemans MJ, Takkenberg JJ. Actual and actuarial probabilities of competing risks: apples and lemons. Ann Thorac Surg. 2007; $83: 1586-92$

27. Grunkemeier GL, Takkenberg JJ, Jamieson WR, Miller DC. Reporting "actual freedom" should not be banned. J Thorac Cardiovasc Surg. 2008;135: 460-2.

28. Grunkemeier GL, Furnary AP, Wu Y, Wang L, Starr A. Durability of pericardial versus porcine bioprosthetic heart valves. J Thorac Cardiovasc Surg. 2012;144: $1381-6$

Key Words: aortic valve replacement, trifecta, durability, outcomes 
TABLE E1. Follow-up results and details of causes of death at follow-up

\begin{tabular}{lc}
\hline \multicolumn{1}{c}{ Characteristic } & Value \\
\hline 5-y freedom from death (any cause), \%, mean \pm SD & $74.9 \pm 8.5$ \\
5-y freedom from valve-related death, \%, mean \pm SD & $97.8 \pm 0.9$ \\
5-y freedom from SVD, \%, mean \pm SD & $98 \pm 0.9$ \\
5-y freedom reintervention (any type) for SVD, \%, & $98 \pm 0.9$ \\
mean \pm SD & \\
5-y freedom from redo surgery for SVD, \%, mean \pm SD & $98.9 \pm 0.6$ \\
Causes of death at follow-up, $\mathrm{n}$ & \\
Valve-related & \\
Unknown cause & 7 \\
Infective (prosthetic) endocarditis & 2 \\
Cardiac non-valve-related & \\
Heart failure with well-functioning prosthesis & 7 \\
Myocardial infarction & 3 \\
Ventricular arrhythmia & 1 \\
Endocarditis in other valve & 1 \\
Noncardiac & \\
Cancer & 17 \\
Suicide & 4 \\
Trauma & 2 \\
Pulmonary failure & 2 \\
Senility & \\
Atherosclerosis & \\
End-organ failure & 2 \\
\hline
\end{tabular}

$S D$, Standard deviation; $S V D$, structural valve deterioration. 